\title{
Analysis of Brain Function and Classification of Sleep Stage EEG using Daubechies Wavelet
}

\author{
Min Soo Kim, Young Chang Cho ${ }^{1}$, Abibullaev Berdakh² and Hee Don Seo,** \\ Dept. of Mechanical Engineering, Pohang University of Science and Technology (POSTECH), \\ Pohang, Kyongbuk, Korea 790-784 \\ ${ }^{1}$ Dept. of Information and Communications Engineering, Kyungwoon University, \\ 55, Indoek-ri, Sandong-myeon, Gumi, Kyongbuk, Korea 730-739 \\ ${ }^{2}$ Dept. of Electronic Engineering, Yeungnam University, 214-1 Dae-dong, \\ Kyongsan, Kyongbuk, Korea 712-749
}

(Received March 20, 2007; accepted September 3, 2007)

Key words: Daubechies wavelet, sleep electroencephalograms, sleep stages, mental tasks, brain computer interface (BCI), cognitive mental state

Recently, wavelet transforms have been applied to various problems in many fields. In this paper, we propose the application of the Daubechies wavelet to the detection of several important characteristic waves in electroencephalograms (EEGs), which are used to diagnose sleep stages and cognitive mental tasks. Sleep staging is one of the most important tasks in EEG diagnosis. However, it can be subjective as it depends on the doctor's skill and is omit labor-intensive. In this regard, the development of an automatic diagnosis system is imperative in order to reduce the doctor's workload and to provide an accurate quantitative diagnosis of sleep stage EEGs. The method proposed in this paper is an important base for understanding subjects' cognitive state. Experimental results obtained using the implemented system demonstrate that this approach could reduce the doctor's workload and provide an accurate diagnosis of brain functions.

\section{Introduction}

Electroencephalograms (EEGs) are widely used to diagnose the activities, functions, ${ }^{(1-6)}$ epileptic state, ${ }^{(7-9)}$ and functional disorders of the brain, ${ }^{(10)}$ and animal experiments aimed at the functional understanding of each part of the brain and research aimed at communication with patients who have serious physical disabilities such as total paralysis are actively under way. ${ }^{(11-13)}$

As for research on sleep EEGs, while a study that analyzes by stage the changes

*Corresponding author: e-mail: hdseo@yumail.ac.kr 
brought about by the depth of sleep ${ }^{(14)}$ is being conducted, relatively little research has been performed to classify or analyze sleep stages clearly thus far. Although sleep waves are generally classified into five stages and analyzed on this basis, it is extremely difficult for a nonexpert to distinguish them with the naked eye because the characteristics of each sleep stage differ slightly. To analyze such characteristics of sleep EEGs accurately, research has been conducted to quantitatively analyze the frequency of EEGs during each sleep stage. ${ }^{(15-18)}$ With the development of the Mind Switch by Kirkup et al. in 1997, research for determing brain functions and the processes of mental mechanisms that occur in the brain using EEGs has gained considerable attention. ${ }^{(19)}$ In such research, the interpretation of mental mechanisms that occur in the human cognitive process has been attempted using EEGs. ${ }^{(20-23)}$ However, only experimental studies are being conducted at present, without the application of the technology to patients at hospitals. In addition, in many studies, brain computer interfaces (BCIs), ${ }^{(24,25)}$ which enable computers to recognize human wishes through brain function testing, are explored.(26,27) Because it enables the moment-by-moment observation of the brain cognitive process, as opposed to functional magnetic resonance imaging (fMRI)(28) and positron emission tomography (PET), ${ }^{(29)}$ measurement using an EEG is outstanding in terms of temporal resolution. As it is also easy to use, the EEG is extremely widely applied. The EEG analysis method mainly used in hospitals involves EEG specialists finding specific EEGs from EEG records with the naked eye. Consequently, not only expertise but also considerable time and effort are required for simple repetitive tasks involved in the analysis. In addition, due to the subjectivity of the analysis, identical EEG records can yield inconsistent results. The automatic detection of EEGs using computers therefore is indispensable for high objectivity or quantitative analysis. However, experiments relying on this method require expensive special equipment and complex directions for using such equipment must be understood first. Consequently, it is necessary to enable nonprofessionals to test brain functions and to analyze the frequency of analysis with ease using less costly equipment and by developing easier to use programs.

In this paper, methods of analyzing sleep stage EEGs through wavelet transform and multilayer neural networks are described. The signal frequency analysis through wavelet transform involves the use of the Daubechies wavelet transform. ${ }^{(30,31)}$ Among existing mother wavelet approaches, this approach is known for outstanding characteristics in time and frequency domains. From the Daubechies family, db6 was used.

The automatic EEG classification method proposed in this paper is expected not only to allow brain specialists to increase the objectivity of diagnosis through a quantitative analysis of brain waves but also to considerably decrease the time and effort required for diagnosis. It could also be applicable to brain computer interface (BCI) systems, which assess brain functions.

\section{Methods}

\subsection{Characteristics of sleep EEGs}

On the basis of the international classification of sleep as determined from stage EEGs, sleep is largely divided into five stages. Stage 1 is defined as a sleeping state 
where $\alpha$-waves are 50 or less and $\theta$-waves of low amplitude and composite frequency are 50 or more. At this stage, ripple waves are visible. Stage 2 is a sleeping state where a sleep spindle of $12-14 \mathrm{~Hz}$ appears for at least 0.5 in the vertex, or a K-complex appears. A moderate sleep stage (stage 3 ) is a sleeping state where $\delta$-waves measuring at most $2 \mathrm{~Hz}$ and $75 \mathrm{uV}$ account for $20-50 \%$ of the epoch. Stage 4 is a sleeping state where $\delta$-waves account for at least $50 \%$ of the epoch. In the rapid eye movement (REM) stage, various EEGs including $\theta$-waves with low amplitudes of $2-7 \mathrm{~Hz}$ and slow waves appear as do, more rarely, $\alpha$-wave bursts and sawtooth waves. In addition, $\alpha$-waves whose rhythm frequency is less than that of $\alpha$-waves in the waking state by $1-2 \mathrm{~Hz}$ can exist.

In this study, the REM stage was determined using only brain waves. The reference points of the electrodes were established as A1 and $\mathrm{A2}^{(32)}$ in Fig. 1. The EEGs in this study were measured using 16 electrodes (Fp1, Fp2, C3, C4, O1, O2, T3, T4, F3, F4, P3, P4, F7, F8, T5, and T6).

\subsection{Mental task state}

In a BCI system for determining cognitive mental states in real time, computers recognize human will through brain function tests.

The experimental equipment used for determining the subject's cognitive mental state consisted of a monitor, an amplifier, an acquisition system, cap electrodes, and an operator. As for the conditions necessary for measurement, the distance between the monitor screen and the subject was approximately $50 \mathrm{~cm}$, with the subject in a comfortable sitting position. The subject was placed in a place that is unaffected by external environmental conditions, i. e., in a soundproof chamber. Figure 2(a) shows the necessary experimental setup, and Fig. 2(b) shows EEG measurement, in which the subject was stimulated by and made judgment on questions presented on the computer monitor by selecting keys. The EEG acquisition system received data through the

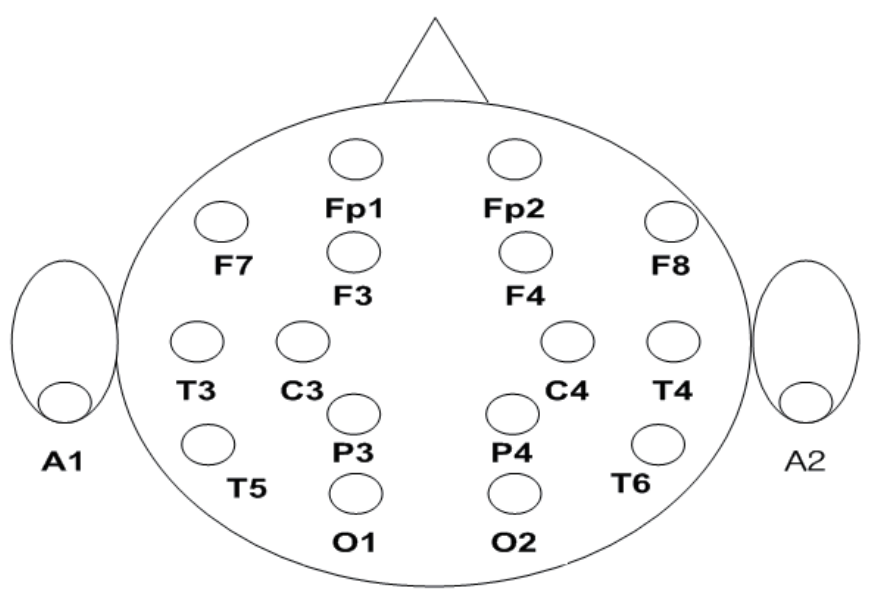

Fig. 1. Position of sleep stage electrodes in 10-20 system. 


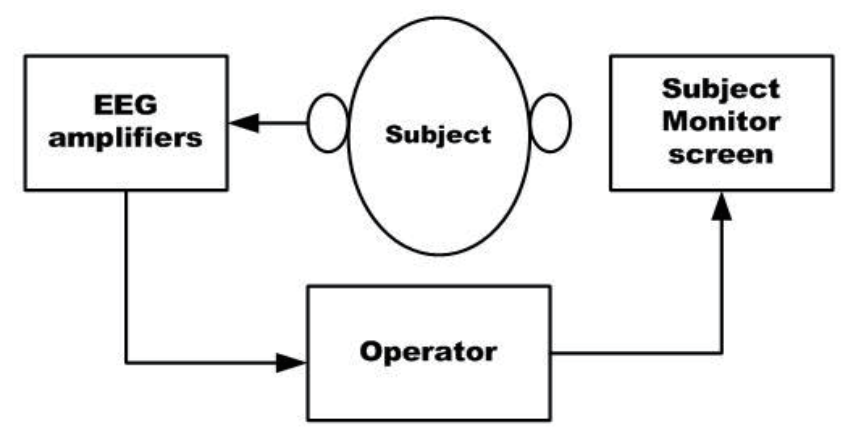

(a)

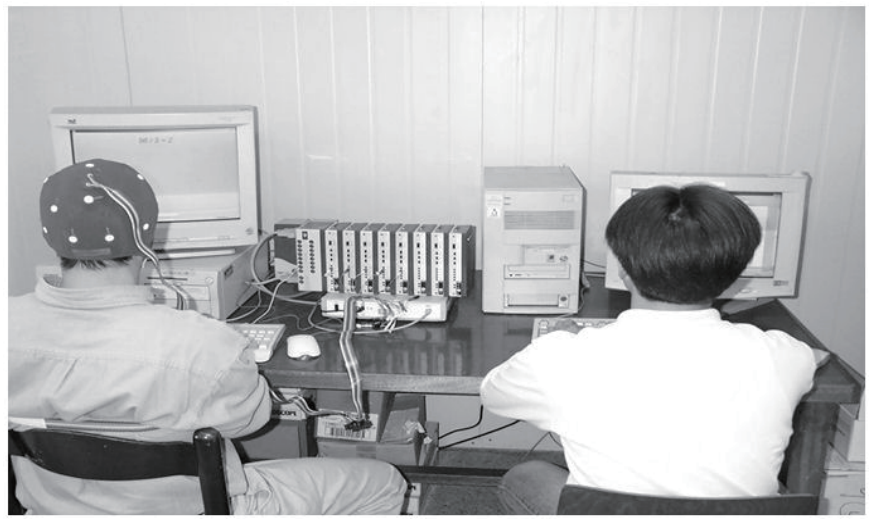

(b)

Fig. 2. Positions of electrodes based on10-20 system. (a) EEG experimental setup. (b) Scene of EEG measurement experiment.

EEG100B amplifier and cap electrodes manufactured by Biopac Systems, Inc. In addition, the operator, who was necessary for the experiment, could observe the subject's brain wave states and control and adjust the devices required for the experiment.

The subject was provided with information on experimental tasks for acculturation.

Various questions were asked to determine changes in brain functions for cognitive activities. Mathematical, Chinese phrase, and color decision tasks were performed by the subject. Each question was presented for 10 on the screen, and during this time, the subject selected his or her answer using three keys. When the response to the content on the monitor screen was affirmative, the subject selected $\mathrm{Y}$ on the keyboard; when it was negative, he or she selected $\mathrm{N}$; and when the subject could not respond, he or she selected the space bar. Correct answers were unavailable during the experiment itself; they were disclosed only afterwards.

The mental tasks described here were constructed using Java programming language. The three tasks investigated in this study included the following:

Baseline measurement: Subjects were asked not to perform a specific mental task but to 
relax as much as possible and to think of nothing in particular. This task was considered the baseline task for $\alpha$-wave production and used as a control measure for EEGs.

Mathematical tasks: Subjects were given calculation problems and asked to solve them without vocalizing or making any other physical movement. The problems were not repeated and were designed so that an immediate answer was not attainable.

Color decision tasks: Subjects were given 10 to discriminate a particular color figure.

Chinese phrase tasks: Subjects were asked to compose mentally a Chinese letter to a relative or a friend without vocalizing.

The subject mental statement task is schematically shown in Fig. 3.

\subsection{Wavelet transformation}

Wavelet analysis methods have been widely employed in signal processing in the biomedical field. $^{(33)}$ In general, these methods represent the temporal characteristics of a signal induced by its spectral components in the frequency domain. A powerful alternative for the analysis of nonstationary signals whose spectral features change over the course of time, that is, wavelet analysis, is very important for the analysis of biological signals, because most of the statistical characteristics of these signals are nonstationary.

Wavelet transformation uses the mother function to disintegrate the original signal into frequency bands with multiresolutions and classifies them into approximations and details. Discrete wavelet transformation consists of scaling functions $\varphi$ and $\Phi$, as in eq. (1).

$$
\begin{gathered}
\varphi(k)=\sum_{n} h(n) \sqrt{2 \varphi(2 k-n)} \\
\phi(k)=\sum_{n} g(n) \sqrt{2 \phi(2 k-n)}
\end{gathered}
$$

The coefficient of $h(n)$ the wavelet filter is the high-pass filter coefficient, and the scaling filter coefficient $g(n)$ is the low-pass filter coefficient. First, the high-pass filter $g(n)$ and low-pass filter $h(n)$, whose bands are one-half that of the input signal $x(n)$, disintegrate the original signal into two frequency bands. Through this process, disintegration at each stage occurs, which can be expressed as eq. (2).

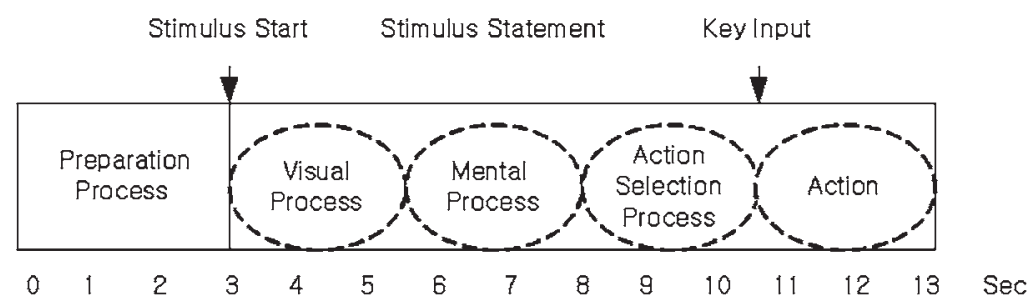

Fig. 3. Analysis of subject mental state. 


$$
\begin{aligned}
c_{j}(k) & =\sum_{n} h(n-2 k) x(n) \\
d_{j}(k) & =\sum_{n} g(n-2 k) x(n)
\end{aligned}
$$

Here, $c_{j}$ represents the original signal after it has passed through the high-pass filter, and $d_{j}$ represents the original signal after it has passed through the low-pass filter. When the original signal is disintegrated with high- and low-pass filters, as in eq. (2), it is expressed as one-half of the number of samples. Consequently, the time resolution is reduced by one-half, thus making down sampling and disintegration into the next stage without information loss possible.

As in Fig. 4, the wavelet function has a short wavelength in high-frequency bands and a long wavelength in low-frequency bands, thus sensitively responding to small signal changes to the signal. Such characteristics are useful for finding the starting point of signal changes in signal processing and can also be used in geometric analysis. On the basis of the characteristics described above, wavelet transformation is considered a signal processing tool that allows the time-frequency analysis of signals and is useful for understanding the physical significance of signals through the analysis of diverse types of signal.

\section{Experiments and Results}

\subsection{Classification system using neural network}

Back-propagation learning (BP) ${ }^{(34,35)}$ paved a way to various applications of neural networks. It has prevailed all over the world and proved the capabilities of the new information processing technology based on neural networks.

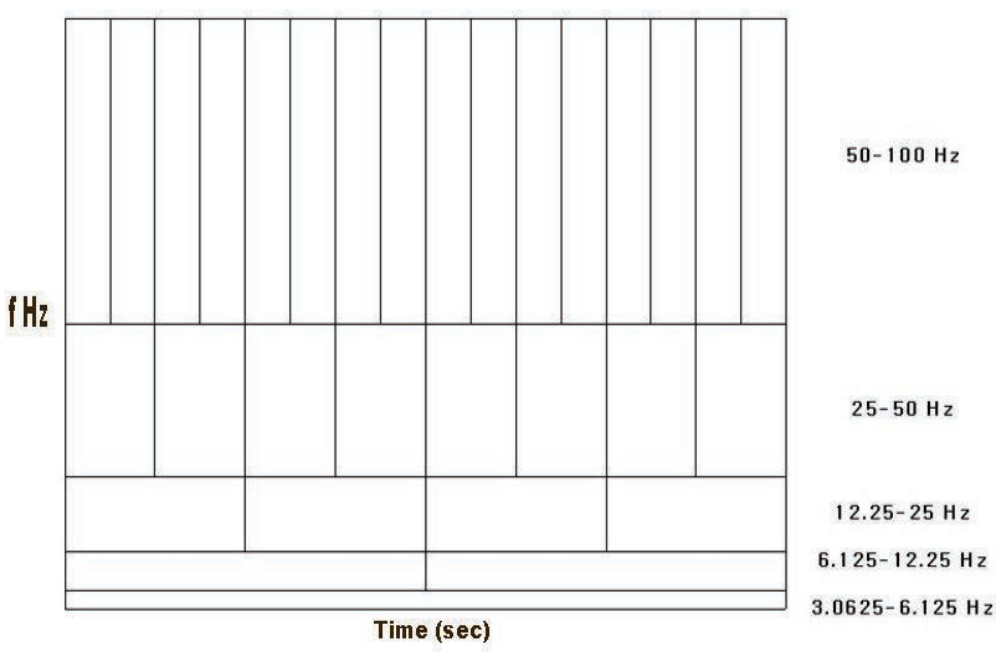

Fig. 4. Time-frequency domain window of wavelet. 
The present implemented neural network achieves learning in three stages using back-propagation algorithms as feedforward multilayer neural networks.

Learning pattern pairs are then entered consecutively to modify synaptic strengths. Because the sigmoid function is used as the activation function, the weighted sum $N E T_{z}$ of the hidden layer, the weighted sum $N E T_{y}$ of the output layer $z$, and the final output $y$ are obtained, as represented by

$$
\begin{gathered}
N E T_{Z}=x V^{T} \\
Z=f\left(N E T_{Z}\right) \\
=\frac{1}{1+\exp \left(1-N E T_{z}\right)} \\
N E T_{y}=z w^{T} \\
y=f\left(N E T_{y}\right) \\
=\frac{1}{1+\exp \left(-N E T_{y}\right)}
\end{gathered}
$$

On the other hand, the square error of the output layer regarding a specific learning pattern is defined as in eq. (9). Equation (10) for the square error $E$ is obtained by comparing the above-mentioned target value $d$ and final output $y$ :

$$
\begin{gathered}
E=\frac{1}{2} \sum_{i=1}^{m}\left(d_{i}-y_{i}\right)^{2} \\
E=\frac{1}{2}(d-y)^{2}
\end{gathered}
$$

Equation (11) shows the error signal $\delta_{y}$ of the output layer, and eq. (12) shows the error signal $\delta_{z}$ transmitted from the hidden layer.

$$
\begin{gathered}
\delta_{y}=(d-y) y(l-y) \\
\delta_{z}=f^{\prime}\left(N E T_{z}\right) \sum_{i=0}^{m} \delta_{y} w
\end{gathered}
$$

$\Delta w^{k}$, which is the change in synaptic strength between the hidden layer and the output layer at the learning stage $k$, and $\Delta v^{k}$, which is the change in the synaptic strength between the input layer and the hidden layer, are then obtained using

$$
\Delta w^{k}=\alpha \delta_{y} z, \quad \Delta v k=\alpha \delta_{z} X
$$


$w^{k+1}$, which is the synaptic strength between the hidden layer and the output layer at the learning stage $k+1$, and $v^{k+1}$, which is the synaptic strength between the input layer and the hidden layer, are obtained using

$$
\begin{gathered}
w^{k+1}=w^{k}+\Delta w^{k} \\
=w^{k}+\alpha \delta_{\mathrm{y}} z^{k} \\
v^{k+1}=v^{k}+\Delta v^{k} \\
=v^{k}+\alpha \delta_{z} x^{k}
\end{gathered}
$$

Here, the synaptic strength is modified by entering learning pattern pairs, and learning is terminated if the error $E$ is smaller than the range of 0.001 . As has been examined above, the BP algorithm is the most frequently used learning theory despite its complex form.

Neural networks into which wavelet coefficients were entered classified sleep stages into $00,01,10$, and 11 . Learning was thereupon implemented and the output was obtained. The maximum number of learning sessions in the neural networks was set at 2,500, and 0.001 was established as the standard for the mean square error.

\subsection{Data acquisition of sleep EEGs}

Sleep was classified into and analyzed in terms of five stages by considering changes in sleep EEGs. For a detailed analysis of sleep stages, the characteristics of each sleep stage were analyzed with the D6 levels of the wavelet.

The data used in the EEG analysis were obtained through clinical experiments at a university hospital and judged by an EEG expert. The harmonic system, manufactured by Stellate Systems, Inc., was used for collecting sleep EEGs. The EEGs were collected from 16 channels, and the frequency bands of the IIR and notch filters were filtered with frequency ranges of $0.5-25 \mathrm{~Hz}$ and $57-63 \mathrm{~Hz}$, respectively. The sampling time was 200 $\mathrm{Hz}$.

Figure 5 shows of sleep EEGs the wavelet decomposition. The EEGs were divided according to eight-stage frequency bands. In other words, discrete wavelet transformation was used to divide EEG signals into signals that had passed through the low-pass filter (A1, A2, A3, A4, A5, A6, A7, and A8) and those that had passed through the high-pass filter (D1, D2, D3, D4, D5, D6, D7, and D8). A1-A8 are low-frequency components that represent the original uncertain signal form, and D1-D8 are highfrequency components that represent the characteristics and energy of the signals in highfrequency bands.

Figure 6 shows the results of the analysis of frequency band signals using a high filter level (D6) that employed wavelet transform. From these results, signal patterns were observed to be similar for stage 1 sleep and REM.

The data used in this experiment were based on the results of an experiment conducted on normal subjects at a university hospital. The EEG data used in neural networks were the sleep waves of subjects aged 35, 25, 30, 40, 7, 13, and 9. All subjects were judged to have normal EEGs. 

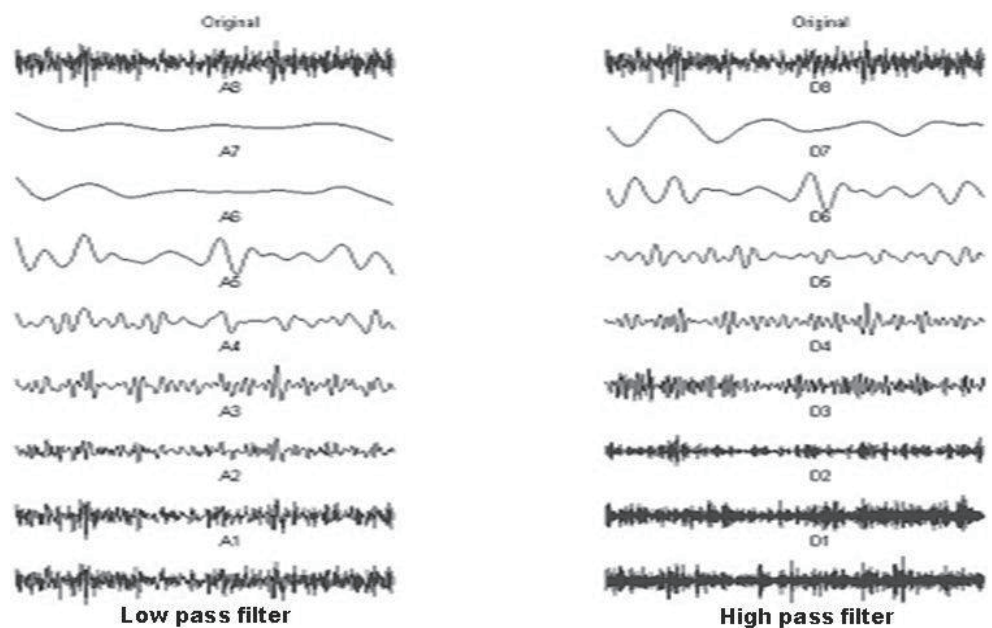

Fig. 5. Wavelet decomposition of sleep EEGs.

Table 1 represents EEGs data at sleep stages entered into the system for classifying sleep stages. Here, age, gender, and the number of sampled data are represented. Table 2 shows the results of an experiment conducted using the characteristic waves of each sleep stage as input signals. In neural networks, the target value $d$ and the output $y$ are compared to obtain the square error rate $E$ and to represent the number of iterations for learning.

Here, the performance of a neural network is expressed in terms of the number of repetitions for learning. The number of repetitions according to the learning rates of stage 4 sleep and REM was recorded nearly 20,000 times and compared with those of the other sleep stages. The results were found to be not entirely favorable. As Table 2 shows, for each sleep stage experiment in terms of the number of repetitions regarding the average equal error rate (EER), learning occurred at an average EER that amounted to a value before the decimal point in the 5,000th session and ended before the 25,000th session. These results were derived from classification experiments after each pattern had been analyzed. Generally, these results were satisfactory, accounting for at least $90 \%$ of classification rate. In contrast, the recognition rate was omit unfavorable in stage 4 sleep and REM. Classification experiments confirmed that cases with a high learning rate likewise yielded satisfactory results on classification performance.

\subsection{EEG analysis during performance of cognitive mental tasks}

This study used subjects' responses to three types of task. To determine cognitive mental states during the performance of each task, EEG signals were obtained, analyzed, and classified. The subjects were mainly undergraduate and graduate students.

This study also used international 10-20 system electrodes, and the standards for frequency bandwidth were $8-13 \mathrm{~Hz}$ for $\alpha$-waves, $14-30 \mathrm{~Hz}$ for $\beta$-waves, $4-7 \mathrm{~Hz}$ for 


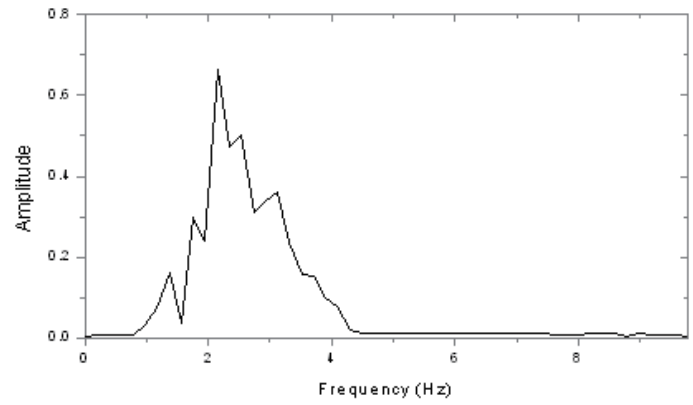

Sleep stage 1 D6

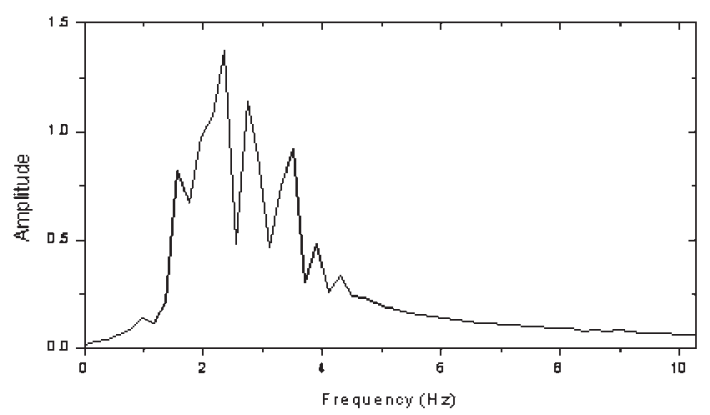

Sleep stage 3 D6

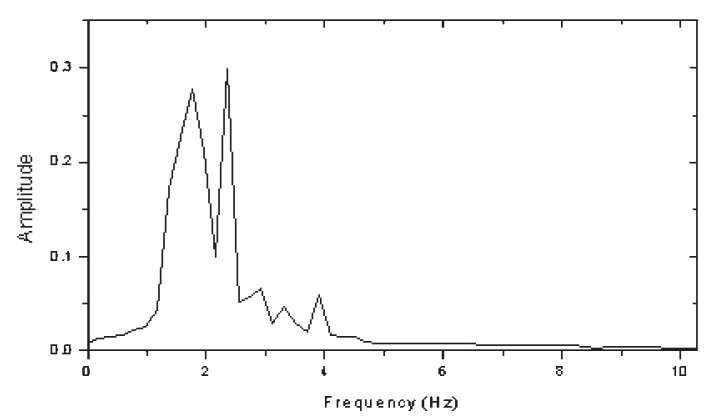

REM stage D6

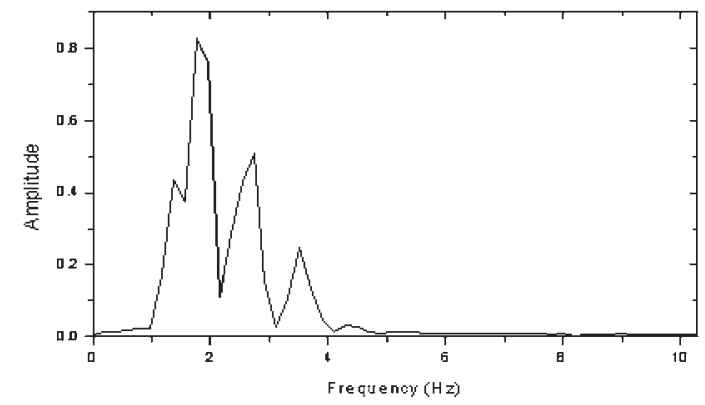

Sleep stage 2 D6

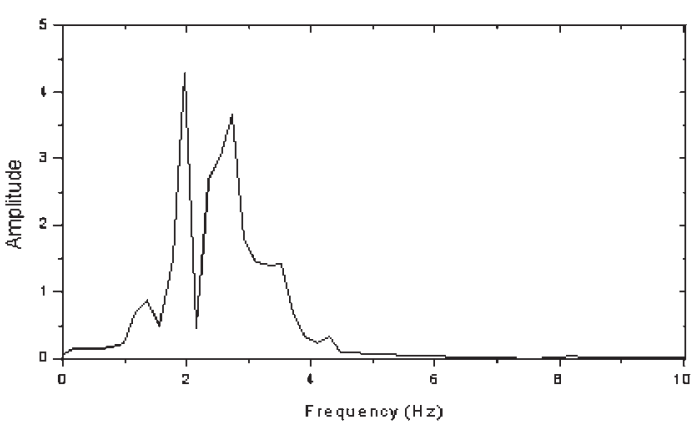

Sleep stage 4 D6

Fig. 6. Analysis of signal levels for sleep stage EEGs.

$\theta$-waves and $31-50 \mathrm{~Hz}$ for $\gamma$-waves. Shifts in EEGs transmitted through cap electrodes attached to the scalp were sampled using the data acquisition system (DAS) at 200 $\mathrm{Hz}$ and simultaneously appeared on the researchers' computer and were recorded in the memory. In the DAS, analog EEGs were transformed into digital signals through processes including the application of an analog-to-digital converter (ADC), an amplifier, filtering, and serial data transport, and were sent to the computer. 
Table l

Subject data used in experiments.

\begin{tabular}{llll}
\hline No. & Age & Sex & Data \\
\hline 1 & 35 & Male & 8286 \\
2 & 25 & Female & 7357 \\
3 & 30 & Male & 9500 \\
4 & 40 & Female & 8800 \\
5 & 7 & Male & 7500 \\
6 & 13 & Female & 8500 \\
7 & 9 & Female & 8900 \\
\hline
\end{tabular}

Table 2

Results of neural network experiment.

\begin{tabular}{lll}
\hline Input & Square error rate & Iteration $(\mathrm{K})$ \\
\hline 1st stage & 0.4923 & 14.3 \\
2nd stage & 0.6123 & 16.6 \\
3rd stage & 0.3395 & 17.5 \\
4th stage & 0.7558 & 18.7 \\
REM & 0.3434 & 19.7 \\
Mean & 0.5086 & 17.36 \\
\hline
\end{tabular}

The EEGs were used to classify mental states by observing and determining the tasks on the subject monitor. Before classifying the detected EEGs, the statistical values of the three types of EEG from each task state were analyzed. In Fig. 7, an analysis of the frequency domain shows the occurrence of $\alpha$-waves in the baseline state in Fig. 7(b) and that of $\beta$-waves in the cognitive mental states in Figs. 7(c)-7(f) frontal lobe $\beta$-waves could be identified more exactly than those in Figs. 7(g) and 7(h). Consequently, it was easy to distinguish between the baseline and cognitive mental states using the frequency domain. Figure 8 shows the results of time domain analysis conducted on the EEGs to determine differences between left/right hand movements during the performance of tasks. Figure 8(a) shows the EEGs obtained when keys were entered with the left hand in response to cognitive orders, and Fig. 8(b) shows those obtained when keys were entered with the right hand in response to cognitive orders. Although there was not no great disparity between the two in cognitive processes, a detailed frequency analysis revealed some differences.

Table 3 shows the results for subjects' performance of various mental tasks. Although there were slight personal differences, subjects' error rate during the performance of Chinese phrase tasks was higher than those for the other tasks. However, in general, it was possible to classify them at an accuracy rate of at least $90 \%$. 
(a)

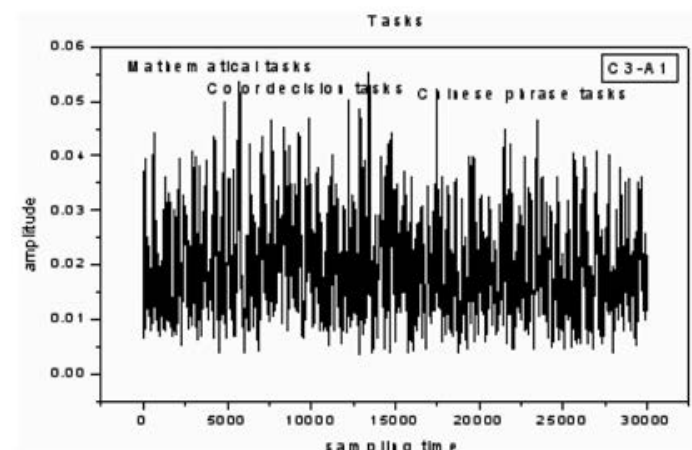

(c)

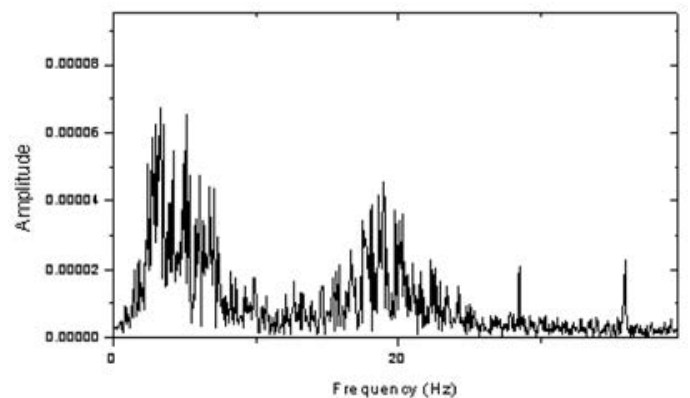

(e)

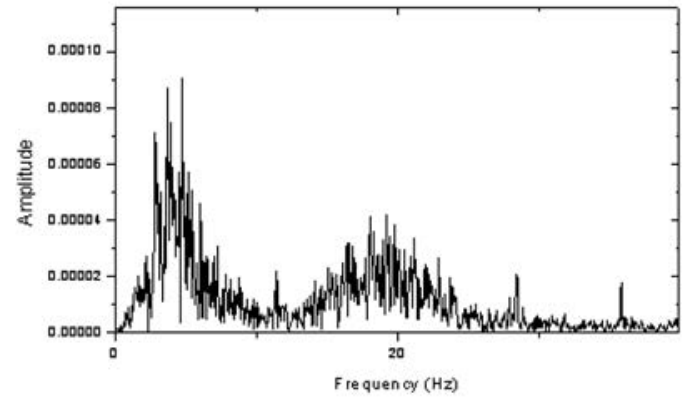

(g)

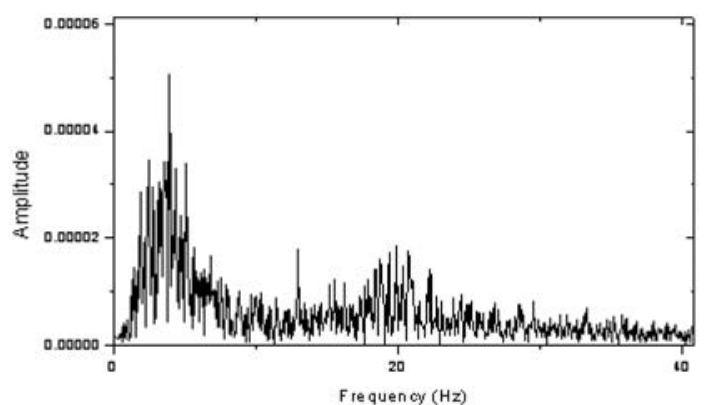

(b)

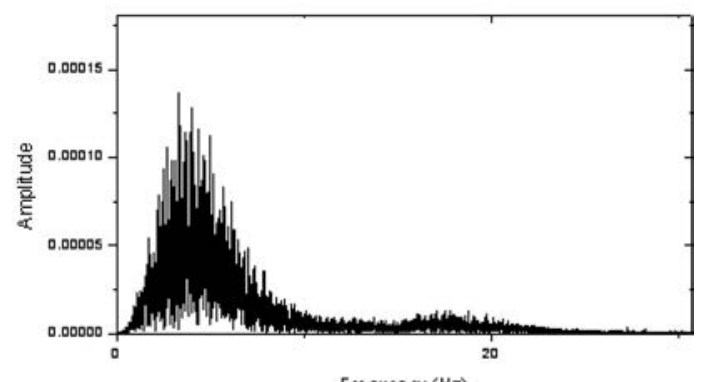

(d)

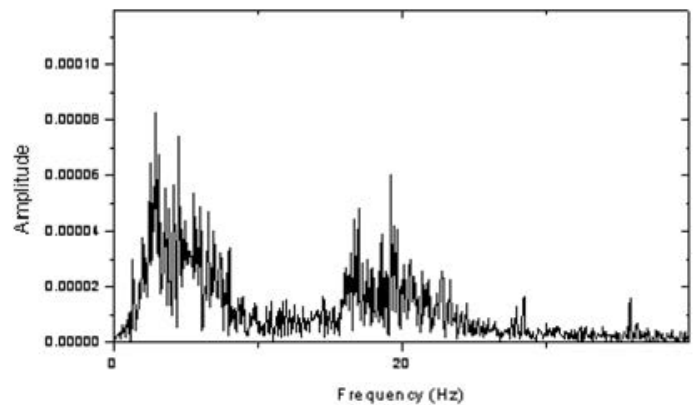

(f)

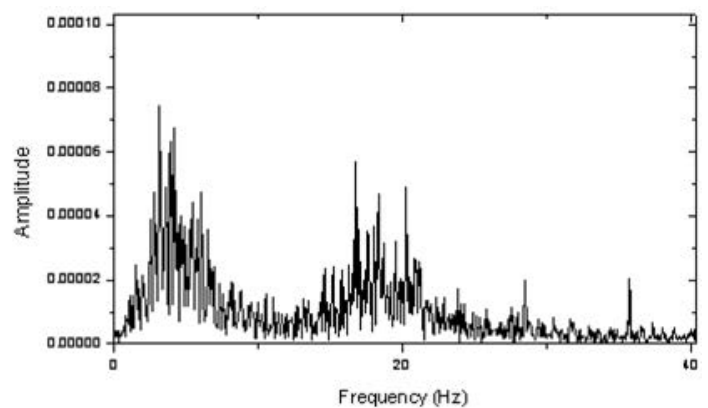

(h)

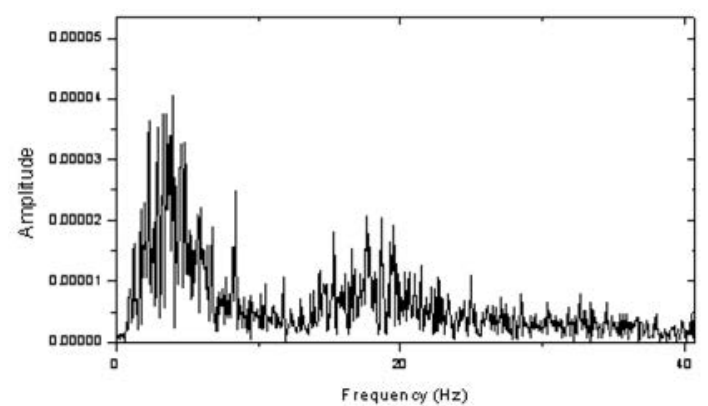

Fig. 7. EEG function during cognitive mental tasks. (a) Time domain, (b) Baseline, (c) Math tasks, (d) Color decision tasks, (e) Chinese phrase tasks, (f) Fp1, (g) O3, and (h) P3. 


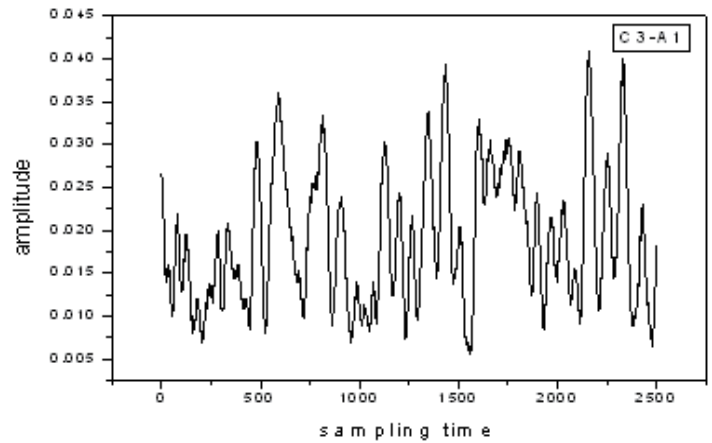

(a)

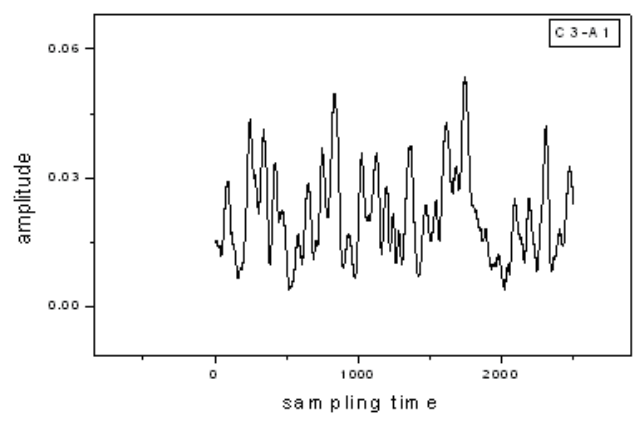

(b)

Fig. 8. EEG comparisons for hand movement by mental tasks. (a) Left, (b) right.

Table 3

Results of BCI experiment.

\begin{tabular}{llll}
\hline Subject & Input result & Test total/wrong & Time ave. (sec) \\
\hline MS & Math. tasks & $10 / 0$ & 6.2 \\
KY & Chinese phrase tasks & $10 / 2$ & 7.1 \\
KM & Math. tasks & $10 / 1$ & 6.3 \\
SM & Math. tasks & $10 / 0$ & 6.4 \\
\hline
\end{tabular}

\section{Conclusion}

In this study, we analyzed human sleep EEGs and EEG characteristics during the performance of cognitive mental tasks by wavelet and fast Fourier transform methods, and proposed a classification system.

The method of frequency division analysis through wavelet transform and classification using neural networks proposed in this study is expected to be useful for EEG experts because it can reduce analysis time and enhance the ability to distinguish EEG signals.

The analysis of EEGs during the performance of cognitive mental tasks was performed by constructing a BCI system. The states in which the subjects reacted were analyzed using the subjects' EEG information to determine the subjects' mental states during the performance of tasks and to verify brain functions. The method proposed in this study is important for understanding subjects' cognitive states.

\section{References}

1 M. Seecka et al.: Electroen. Clin. Neurophysiol. 106 (1998) 508.

2 F. Lazeyras et al.: J. Magn. Reson. Imaging 13 (2001) 943.

3 C. Phillips, M. D. Rugg and K. J. Friston: NeuroImage 16 (2002) 678.

4 T. Matsuda et al.: Int. Cong. Series 1232 (2002) 351. 
5 F. L. Silva: Magnetic Resonance Imaging 22 (2004) 1533.

6 S. H. Na , S. H. Jin and S. Y. Kim : Int. J. Psy. 62 (2006) 238.

7 A. M. Coenen et al.: Pharmacol. Biochem. Behav. 51 (1995) 353.

8 R. J. Staba, C. L. Wilson, A. Bragin, I. Fried and J. E. Jr: J. Neuro. 22 (2002) 5694.

9 S. M. Testa et al.: Int. Lea. Again. Epil. 48 (2007) 973.

10 L. Gloria and B. Reina: Pharm. Res. 21 (2004) 1313.

11 E. Niedermeyer and F. Da Silva: Electroencephalography: Basic Principle, Clinical Applications, and Related Fields (3rd Williams \& Wilkins, Baltimore).

12 R. E. Isaacs, D. J. Weber and A. B. Schwartz: IEEE Trans. Rehab. Eng. 8 (2000) 196.

13 E. Donchin, K. M. Spencer and R. Wijesinghe: IEEE Trans. Rehab. Eng. 8 (2000) 174.

14 T. Shimada and T. Shiina: IEEE Trans. Biomed. Eng. 47 (2000) 369.

15 J. Prcipe, S. Gala and T. Chang: IEEE Trans. Biomed. Eng. 36 (1989) 503.

16 H. D. Seo and M. D. Kim: World Congress on Medical Physics and Biomedical Engineering (Sydney, Australia, 2003) p. 890.

17 B. Bjorbatn, S. Gerland and R. Ursin: Physiol. Behav. 63 (1998) 413.

18 S. V. Argyropoulos et al.: Psychiatry Research 120 (2003) 179.

19 L. Kirkup, A. Searle and A. Craig: Med. Biol. Eng. Comp. 35 (1997) 504.

20 C. W. Anderson, E. A. Stolz and S. Shamsunder: IEEE Trans. BME 45 (1998).

21 A. Schloegl, B. Schack, G. Florian, K. Lugger, M. Pregenzer and G. Pfurtscheller: A Comparison of Different Parameters, TM (Graz University, Austria, 1966).

22 G. Pfurtscheeler, Ch. Neuper, D. Flotzinger and M. Pregenzer: Electro. Clin. Neure. 103 (1997) 642.

23 M. J. Kim, S. C. Shin, Y. S. Song and C. S. Ryu: Proc. IEEE Eng. Med. Bio. Society (Istanbul, Turkey, 2001) 25.

24 J. R. Wolpaw et al.: Clin. Neuro. 113 (2002) 767.

25 T. Hinterberger et al.: Clin. Neuro. 114 (2003) 416.

26 G. Muller and C. Neuper: IEEE Trans. Neu. Sys. Reh. Eng. 11 (2003) 54.

27 C. Ming, G. Xiaorong and G. Hangkai: IEEE Trans. BME. Eng. 49 (2002) 1181.

28 Z. Liu, F. Kecman and B. He: Clin. Neuro. 117 (2006) 1610.

29 K. R. Alper et al.: Psychiatry Res. Neuroima. 146 (2006) 271.

30 M. Vetterli and C. Herley: IEEE Trans. Signal Proc. 40 (1992) 2207.

31 I. Daubechies: Comm. Pure Appl. Math. (1988) 909.

32 F. Patricia and S. Theda: Grass Instrument Company (1974).

33 M. Akay: Annals of Biomed. Eng. 23 (1995) 531.

34 R. K. Brouwer: Neurocomput. 16 (1997) 117.

35 V. Khandetsky and I. Antonyuk: NDT\&E International 35 (2002) 483. 